\title{
Throughput and Delay Analysis of Wireless Random Access Networks
}

\author{
Lin Dai ${ }^{1}$ and Tony T. Lee ${ }^{2}$ \\ 1. Department of Electronic Engineering, City University of Hong Kong \\ 2. Department of Information Engineering, Chinese University of Hong Kong
}

\begin{abstract}
The network throughput and transport delay of a wireless random access network are studied in this paper based on a Markov renewal model of packet transportation. We show that the distribution of the source-to-destination (SD) distance plays a crucial role in characterizing the network performances. The necessary and sufficient condition on the SD-distance is established for scalable network throughput. The optimal rate allocation issue is also addressed with fairness taken into consideration. In order to achieve the network scalability, several traffic scaling laws are delineated by localizing the traffic pattern, and a leaky bucket scheme at the network access is proposed for traffic shaping and flow control.
\end{abstract}

\section{INTRODUCTION}

The link capacity of a point-to-point wireless channel is usually characterized by the effects of noise and fading. In a multi-hop wireless network with many concurrent transmissions, the network capacity is influenced by additional factors such as interference and distance of packet transportation. The interference issue has been extensively investigated in the context of multiuser information theory [1] and multi-access communications [2-3]. Despite the encouraging progress on establishing the capacity regions of broadcast/multi-access channels as well as simple relay channels, extension of these approaches to an arbitrary $n$-node multi-hop network has encountered tremendous difficulties. At the same time, treating interference as contention, numerous random-access protocols have been proposed for wireless networks. Most of these studies, however, focus only on small networks where all nodes are in each other's interference range. In a large-scale wireless network, each node has a finite interference range beyond which other nodes can transmit concurrently. Thus, the contention issue here is different from that in the traditional ALOHA systems because of the spatial reuse that allows concurrent transmissions if the distances between the respective transmitter-receiver pairs are sufficiently large.

Packet transportation is another fundamental issue that affects the network throughput in wireless networks. This issue did not draw much attention until the emergence of wireless ad-hoc networks [4]. In contrast to cellular systems, nodes not only generate their own packets, but also relay others' packets in wireless ad-hoc networks. The transport capacity per node can be roughly expressed as $\theta=\lambda \mathrm{E}[L]$, where $\lambda$ is the input rate (packet per unit time) of each node, and $L$ is the source-todestination (SD) distance, or the number of hops. Given the fact that the transport capacity is limited by the local node throughput, the heavy burden of transport load without any restraints on the SD distance $L$ may eventually drag the network throughput down to zero.

The preceding issues were first addressed by Gupta and Kumar in [5], which embarked on extensive studies of the throughput of wireless networks. In the random network model proposed in [5], the key assumption is that each node randomly picks a destination node with equal probability among all nodes in the network. As a result, the number of possible destination nodes would grow linearly with respect to the SD distance $L$, and the source node is more likely to pick a faraway destination node than a close one. Under this assumption, the achievable network throughput scales as $\Theta(1 / \sqrt{n \log n})$, where $n$ is the total number of nodes in the network. A less pessimistic scaling law $\Theta(1 / \sqrt{n})$ was observed in [6-7] using a similar network model. These results conclude that the network throughput will approach zero with an increasing $n$. This conclusion was further elaborated and verified in a series of follow-up papers [8-11]. More sophisticated models were also developed to incorporate node mobility or fading [12-17] -they were obviously inspired by this rather pessimistic result and aimed at improving the network throughput. As mentioned earlier, the input rate $\lambda$ and the SD distance $L$ are intrinsic elements affecting the network throughput. The key to scalable throughput lies in (i) the local access-protocol operation at each node; and (ii) the global condition on the distribution of the SD distance $L$.

The large amounts of intertwined interactions among distributed nodes make it difficult to model a dynamic wireless network (compared with a wired network) to capture all traffic characteristics. Since the network performance depends mainly on the interference and SD distance distribution, a statistical modeling approach is more appropriate. In our statistical model, $n$ nodes are uniformly distributed over an area and each node can successfully deliver a packet only if there is no other concurrent transmission within the interference range of the receiver. Furthermore, we assume that all source nodes comply with the same SD distance distribution in selecting their destination nodes. The packets are forwarded to the next hop by following the minimum Euclidean distance route. The network throughput is defined to be the equilibrium throughput of each node with conservation of the overall traffic flow taken into account.

In light of the concerns and assumptions discussed above, a Markov renewal process is constructed to model the packet transportation behavior, which enables us to explore several 
key network characteristics. We show that the local access protocols do not affect the order of the network throughput, but the distribution of the SD distance does. We establish the necessary and sufficient condition on the SD distance for scalable network throughput and demonstrate that scalable network throughput can be achieved by localizing the traffic pattern to bind the transport load as the number of nodes $n$ increases. Based on this necessary and sufficient condition, we suggest three sets of traffic scaling laws and propose a leaky bucket scheme to illustrate how to execute the traffic shaping and flow control over the network. The optimal rate allocation issue is also addressed with fairness taken into consideration.

Yet another frontier explored by us is the delay performance. Most prior studies on the network performance focus on the transport capacity. In practical consideration of actual networks, the "transport delay" is also an important performance measure - high transport capacity obtained at the expense of excessive transport delay may not be acceptable for many applications. The study of delay distribution is therefore critical. The distribution of transport delay can be derived from our statistical network model in a straightforward manner. The combination of per-hop delay distribution and the scalable SD distance distribution provides us a coherent landscape of the overall wireless network performance.

The remainder of this paper is organized as follows. Section II is devoted to the modeling of packet transportation which lays the foundation of the whole paper. Section III and IV focus on detailed analyses on network throughput and traffic scaling laws, respectively. Finally, Section V summarizes and concludes this paper.

\section{PACKET TRANSPORTATION MODEL}

Consider a homogeneous and ergodic wireless network with $n$ nodes uniformly distributed in an area $A$. Suppose that the node density $\sigma=n / A$ keeps constant and all nodes comply with the same distribution in selecting their destinations in an isotropic manner. Packets are forwarded to the destinations over multiple hops by following the minimum Euclidean distance route. Let $L$ be the SD-distance (in unit of number of hops) of a newly-generated packet, which takes values in the sample space $S_{L}=\{1,2, \ldots, \varphi\}$. Let $\lambda(l)$ be the average input rate of the newly-generated packets with $l$ SD-hops at each node, and $\lambda=\sum_{l=1}^{\varphi} \lambda(l)$ is the total input rate of each node. The probability mass function of $L$ is then given by

$$
f_{L}(l)=\left\{\begin{array}{cc}
\lambda(l) / \lambda & l \in S_{L} \\
0 & l \notin S_{L}
\end{array}\right.
$$

where $\lambda(l)$ is the average input rate of the newly-generated packets with $l \mathrm{SD}$-hops at each node, and $\lambda=\sum_{l=1}^{\varphi} \lambda(l)$ is the total input rate of each node.

Assume the random access protocol is adopted, such that each node can successfully transmit a packet only if there are no other concurrent transmissions inside the interference range of the receiver. The per-hop delay, including queueing delay and transmission delay, of each packet $\left\{T_{i}\right\}_{i \in S_{L}}$ are i.i.d. random variables with a common probability mass function, $f_{T}(t)$.
The SD-distance $L$ and the per-hop delay $T$ are two pivotal factors characterizing the packet transportation behavior of each single packet. It is clear that $T$ is determined by the local access protocol and $L$ depends on the global conditions such as routing and selection strategies of destinations. A Markov renewal process will be constructed to model the packet transportation and its potency for network performance analysis will be demonstrated.

Assume time is slotted with integer units $t \in\{0,1,2, \ldots\}$. The transition of a tagged packet takes place only when the packet is successfully forwarded. Let $X_{k}$ denote the residual number of hops that the packet must travel after its $k$-th transition, and let $J_{k}$ denote the epoch at which the $k$-th transition occurs. The stochastic process $(\boldsymbol{X}, \boldsymbol{J})=\left\{\left(X_{k}, J_{k}\right), k=0,1, \ldots\right\}$ is then a timehomogeneous discrete Markov renewal process with state space $S_{L}$, transition probability matrix of the embedded Markov chain $\mathbf{P}=\left\{p_{i j}\right\}$, and holding time distributions $\left\{G_{i j}(t)\right\}$, where $p_{i j}=\operatorname{Pr}\left\{X_{k+1}=j \mid X_{k}=i\right\}$ and $G_{i j}(t)=\operatorname{Pr}\left\{J_{k+1}-J_{k} \leq t \mid X_{k+1}=j, X_{k}=i\right\}$.

The transition probability matrix $\mathbf{P}$ is determined by the routing strategy and the probability mass function of the SDdistance $L$. We assume that the packet is always forwarded to the next hop by following the shortest route, and the network is saturated such that as soon as the tagged packet reaches its destination, a new packet will be injected into the network. It is easy to see that in this case the state of the packet is always decremented by one until it reaches State 1. Then the transportation process of the tagged packet will renew at the next transition, and it will jump to State $l$ with probability $f_{L}(l)$ for some $l \in S_{L}$. The transition probability matrix $\mathbf{P}$ of the embedded Markov chain is therefore given by

$$
\mathbf{P}=\left[\begin{array}{ccccc}
f_{L}(1) & f_{L}(2) & \cdots & f_{L}(\varphi-1) & f_{L}(\varphi) \\
1 & & & & \\
& 1 & & & \\
& & \ddots & &
\end{array}\right]
$$

and the limiting probabilities of the embedded Markov chain can then be obtained as

$$
\pi_{l}=\frac{1}{\mathrm{E}[L]} \sum_{i=l}^{\varphi} f_{L}(i), l=1, \ldots, \varphi .
$$

Before a transition takes place, the sojourn time in any given state $l$ is the per-hop delay $T_{l}$ with the probability mass function $f_{T}(t)$. Therefore, the holding time distributions are given by

$$
G_{i j}(t)=\left\{\begin{array}{cc}
F_{T}(t) & i-j=1 \text { or } i=1 \\
0 & \text { otherwise }
\end{array}\right.
$$

and the mean holding time in state $l$ is

$$
\tau_{l}=\sum_{i=1}^{\varphi} p_{l i} \tau_{l i}=\mathrm{E}[T], \quad l=1, \ldots, \varphi .
$$

where $\tau_{l i}=\int_{0}^{\infty} t d G_{l i}(t)$ is the mean holding time in state $l$ conditional on the destination state $i$.

The limiting probabilities of the Markov renewal process $(\boldsymbol{X}$, $\boldsymbol{J}), \tilde{\pi}_{l}, l=1, \ldots, \varphi$, can be finally obtained from (3) and (5) as [18]:

$$
\tilde{\pi}_{l}=\frac{\pi_{l} \tau_{l}}{\sum_{i=1}^{\varphi} \pi_{i} \tau_{i}}=\pi_{l}=\frac{1}{\mathrm{E}[L]} \sum_{i=l}^{\varphi} f_{L}(i)
$$


It is clear from (6) that the long-run fraction of time in any state $l, \tilde{\pi}_{l}$, is only determined by the distribution of SDdistance, $f_{L}(l)$, due to the identical mean holding time in state $l$.

The Markov renewal process $(\boldsymbol{X}, \boldsymbol{J})$ enables us to explore various properties of packet transportation. Define $\widehat{L}(t)$ as the residual number of hops of a packet at time $t$. We have

$$
\widehat{L}(t)=X_{k}, \quad J_{k} \leq t \leq J_{k+1} \text {. }
$$

It is easy to see that the stochastic process $\widehat{\boldsymbol{L}}=\{\widehat{L}(t), t=0,1, \ldots\}$ is a semi-regenerative process in which the Markov renewal process $(\boldsymbol{X}, \boldsymbol{J})$ is embedded. Fig. 1 presents a sample path of $\widehat{\boldsymbol{L}}=\{\widehat{L}(t), t=0,1, \ldots\}$.

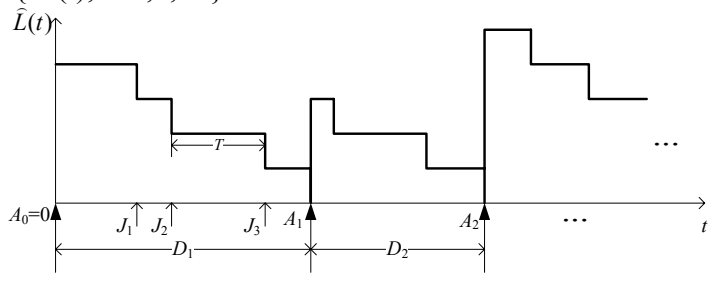

Fig. 1. A sample path of $\{\widehat{L}(t), t=0,1, \ldots\}$.

The limiting probabilities of $\hat{\boldsymbol{L}}$ are given by [18]

$$
\lim _{t \rightarrow \infty} \operatorname{Pr}\{\hat{L}(t)=l\}=\tilde{\pi}_{l}, l=1, \ldots, \varphi .
$$

From (6) and (8), we immediately obtain the steady-state moment generating function of $\widehat{\boldsymbol{L}}$ as follows:

$$
M_{\bar{L}}(z)=\sum_{l=1}^{\infty} z^{l} \cdot \tilde{\pi}_{l}=\frac{1}{\mathrm{E}[L]} \sum_{l=1}^{\varphi} f_{L}(l) \sum_{k=1}^{l} z^{k}=\frac{z\left(1-M_{L}(z)\right)}{(1-z) \mathrm{E}[L]} .
$$

Since the network is ergodic, the mean residual number of hops per packet should be the same as that of the tagged packet:

$$
\mathrm{E}[\widehat{L}]=\left.\frac{d M_{\bar{L}}(z)}{d z}\right|_{z=1}=\frac{\mathrm{E}\left[L^{2}\right]+\mathrm{E}[L]}{2 \mathrm{E}[L]}
$$

Note that the mean residual number of hops given in (10) is dependent on the second moment of $L$. This is the well-known sample bias property of residual life in renewal theory [19]: The packets with larger SD-distance $L$ stay in the network longer and therefore have larger probability being observed.

As shown in Fig. 1, the sequence of regeneration epochs $\left\{A_{0}, A_{1}, A_{2}, \ldots\right\}$ of $\boldsymbol{L}$ form a renewal process, where $A_{i}$ is the $i$ th renewal of the tagged packet. The inter-arrival times, $D_{i}=A_{i}-A_{i-1}$, are i.i.d. random variables with the common probability mass function

$$
\operatorname{Pr}\{D=x\}=\operatorname{Pr}\left\{\sum_{j=1}^{L} T_{j}=x\right\}, \quad x=1,2, \ldots
$$

The inter-arrival time $D$ is referred to as the transport delay of the packet. The moment generating function of $D$ can be obtained immediately from (11):

$$
M_{D}(z)=\mathrm{E}\left[z^{D}\right]=\sum_{l=1}^{\infty} M_{T}^{l}(z) \cdot f_{L}(l)=M_{L}\left(M_{T}(z)\right)
$$

where $M_{T}(z)$ is the moment generating function of the per-hop delay $T$. The first and second moments of transport delay $D$ are therefore given by

$$
\mathrm{E}[D]=\mathrm{E}[L] \cdot \mathrm{E}[T]
$$

and

$$
\mathrm{E}\left[D^{2}\right]=\mathrm{E}\left[L^{2}\right](\mathrm{E}[T])^{2}+\mathrm{E}[L] \operatorname{var}[T]
$$

\section{NETWORK THROUGHPUT}

Based on the packet transportation model described in Sec. II, this section is devoted to the detailed analysis on network throughput. In this paper, the network throughput is defined to be the equilibrium input rate $\lambda$ of each node. Let $N$ be the total number of packets in the network. For given input rate $\lambda$ of newly generated packets at each node and mean transport delay $\mathrm{E}[D]$, the expected number of packets in the network should be equal to

$$
\bar{N}=n \lambda \mathrm{E}[D]
$$

according to Little's law, where $n$ is the number of nodes. On the other hand, the total arrival rate of each node's buffer, denoted by $\theta$, includes both the newly generated packets and the relay packets. Thus, the expected number of packets in each node's buffer is given by $\theta \mathrm{E}[T]$, where $\mathrm{E}[T]$ is the mean perhop delay. Again, from Little's law we have

$$
\bar{N}=n \theta \mathrm{E}[T]
$$

It is known from the throughput analysis of random access protocol that the throughput of each node, which equals the arrival rate $\theta$ in equilibrium, should be bounded by $e^{-1} /\left(\pi R^{2} \sigma\right)$, where $\pi R^{2} \sigma$ is the number of nodes within the interference range of radius $R$. Combining (13), (15) and (16), we establish the following fundamental relationship between the input rate $\lambda$ and the local node throughput $\theta$ :

$$
\lambda \mathrm{E}[L]=\theta \leq e^{-1} /\left(\pi R^{2} \sigma\right)
$$

where the left side of (17) is also called the transport capacity of each node in [5], which includes the packets initiated by the node and the relay packets generated by other nodes. The right side of (17) is the maximum output rate per node under the random access protocol. Eqn. (17) implies the conservation of network flows that the traffic input rate should not exceed the network output rate. In this paper, the network throughput is defined to be the equilibrium input rate of each node, $\lambda$.

Note that the local node throughput $\theta$ is determined by the random access protocol, and it is a constant independent of the SD-distance $L$. The mean SD-distance per packet $\mathrm{E}[L]$ is bounded by the maximum number of SD hops $\varphi$ which has the order of $\varphi \sim \Theta(\sqrt{n})$ in an $n$-node network. According to (17), we know that the network throughput $\lambda \geq \theta / \varphi$. This indicates that the network throughput should at least have an order of $\lambda \sim \Theta(1 / \sqrt{n})$. Note that it has been proved in [7] that the network throughput $\lambda$ also scales as $\lambda \sim \Theta(1 / \sqrt{n})$ even with the optimal scheduling. The fact that the network throughput has the same order performance in the worst (random access) and best (optimal scheduling) scenarios indicates that the local access protocols do not change the order results on the network throughput $\lambda$. Later we will show that better throughput performances, for example $\lambda \sim \Theta(1)$, can only be achieved when the distribution of $L$ satisfies some specific constraints.

Eqn.(17) manifests the tradeoff between the throughput $\lambda$ and the mean number of hops $\mathrm{E}[L]$. Consider the special case $\sum_{l=1}^{M} l f_{L}(l)=0$, which implies $\mathrm{E}[L] \geq M+1$ and therefore $\lambda \leq$ $\theta /(M+1)$. As a result, the network throughput will approach zero with $M$ increasing. This kind of tradeoff suggests that scalable network throughput can not be achieved if fewer and fewer packets are delivered inside each node's proximity as the 
number of nodes $n$ increases. This point will be elaborated in the following subsection.

\section{A. Scalable Network Throughput}

A scalable network throughput requires that $\lambda$ does not approach zero when the number of nodes $n$, or equivalently, the maximum number of SD hops $\varphi$, goes to infinity. The following Theorem presents the necessary and sufficient condition of a scalable network throughput.

Theorem 1. $\lim _{\varphi \rightarrow \infty} \lambda>0$ if and only if there exists some $M \geq 1$ such that $\lim _{\varphi \rightarrow \infty} \sum_{l=1}^{M} l \lambda(l)>0$.

Proof: (1) If: $\lim _{\varphi \rightarrow \infty} \lambda=\lim _{\varphi \rightarrow \infty} \sum_{l=1}^{\varphi} \lambda(l) \geq \lim _{\varphi \rightarrow \infty} \sum_{l=1}^{M} \lambda(l)$ $\geq \lim _{\varphi \rightarrow \infty} \frac{1}{M} \sum_{l=1}^{M} l \lambda(l)>0$.

(2) Only if: We try to prove that if for any $M \geq 1$, $\lim _{\varphi \rightarrow \infty} \sum_{l=1}^{M} l \lambda(l)=0$, then $\lim _{\varphi \rightarrow \infty} \lambda=0$.

For any $\varepsilon>0$, let $a=\lceil\theta / \varepsilon\rceil$. Then $\lambda=\sum_{l=1}^{a} \lambda(l)+\sum_{l=a+1}^{\varphi} \lambda(l)$.

1) From $0 \leq \lim _{\varphi \rightarrow \infty} \sum_{l=1}^{a} \lambda(l) \leq \lim _{\varphi \rightarrow \infty} \sum_{l=1}^{a} l \lambda(l)=0$, we know that $\lim _{\varphi \rightarrow \infty} \sum_{l=1}^{a} \lambda(l)=0$.

2) $\sum_{l=a+1}^{\varphi} \lambda(l)=\sum_{l=a+1}^{\varphi} \frac{1}{l} \cdot l \lambda(l) \leq \theta /(a+1)<\varepsilon$. Therefore, for any $\varepsilon>0$, there exists $a=\lceil\theta / \varepsilon\rceil$ such that $\sum_{l=a+1}^{\varphi} \lambda(l)<\varepsilon$ whenever $\varphi>a$. This means $\lim _{\varphi \rightarrow \infty} \sum_{l=a+1}^{\varphi} \lambda(l)=0$.

By combining 1) and 2), we have $\lim _{\varphi \rightarrow \infty} \lambda=0$.

The above theorem provides the criteria to verify whether a scalable throughput is achievable for a given rate allocation pattern $\lambda(l)$. For example, in [5-11] it is assumed that each node randomly picks a destination node with equal probability among all nodes in the network. This leads to the following rate allocation:

$$
\lambda(l)=\frac{l \lambda_{0}}{\varphi(\varphi+1) / 2}
$$

where $\lambda_{0}$ is a constant. Clearly for any $M \geq 1$, we have

$$
\lim _{\varphi \rightarrow \infty} \sum_{l=1}^{M} l \lambda(l)=\lim _{\varphi \rightarrow \infty} \frac{\lambda_{0} M(M+1)(2 M+1) / 6}{\varphi(\varphi+1) / 2}=0
$$

Therefore, we conclude that no scalable network throughput can be achieved in this case.

Fig. 2 (a) further illustrates the traffic pattern under this equal-probability-selection assumption. Here the probability of the destination being a node at the periphery is always the highest because there are more nodes there. As we can see, this leads to a "vacuum zone" (i.e., the probability of selecting a destination node inside this zone is less than an arbitrary small number $\varepsilon>0$ ) surrounding each source node, and the radius of this vacuum zone will increase with the number of nodes $n$. In other words, in this case the network traffic will be marginalized as the network scale increases and that will eventually drag the network throughput down to zero.

In a scalable network, the traffic should be localized so that the communication area of each node does not increase with the total number of nodes, $n$. As Fig. 2 (b) shows, Theorem 1 requires that the network traffic inside some local area does not diminish as the network grows. This usually can be guaranteed by the so-called "local preference" in practical networks. For example, it is more likely for a person in the New York City to make a telephone call to another person in the New York City than to another person in, say, Hong Kong. This does not change with the network scale and is one of the principles behind organizing a practical network in a hierarchical manner. For that reason, we believe that a more realistic traffic model should take "local preference" into account. Theorem 1 demonstrates that adopting the locality principle of traffic pattern will lead to a more down-to-earth conclusion in network throughput.

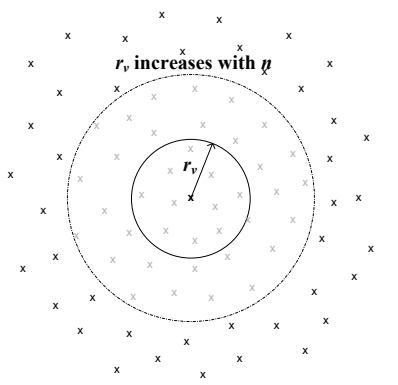

(a)

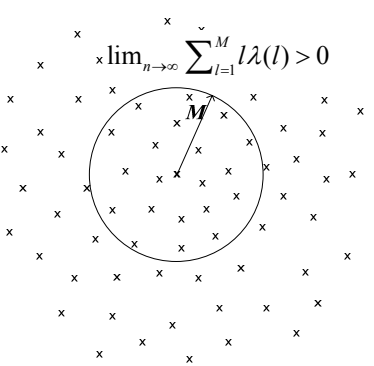

(b)
Fig. 2. (a) Traffic pattern in the equal-probability-selection case. (b) Traffic pattern in a scalable network.

We can also see from (17) that a scalable network throughput $\lim _{\varphi \rightarrow \infty} \lambda>0$ requires that the mean SD-distance is bounded, i.e., $\lim _{\varphi \rightarrow \infty} \mathrm{E}[L]<\infty$. The necessary and sufficient condition provided in Theorem 1 is equivalent to $\lim _{\varphi \rightarrow \infty} \mathrm{E}[L]<\infty$ and both of them can be used as convenient ways to check whether a given rate allocation pattern $\lambda(l)$ can lead to a scalable network throughput. We demonstrate it through the following examples.

Example 1: $\lambda(l)=\lambda_{0} / \varphi$

According to (1), the distribution of SD-distance $f_{L}(l)=1 / \varphi$ and we have

$$
\lim _{\varphi \rightarrow \infty} \mathrm{E}[L]=\lim _{\varphi \rightarrow \infty}(\varphi+1) / 2=\infty .
$$

Therefore, we conclude that the network throughput achieved in this case is non-scalable.

Example 2: $\lambda(l)=\lambda_{0} \omega(l), \omega(l)$ is an arbitrary probability mass function with $\sum_{l=1}^{\varphi_{0}} \omega(l)=1$, and the maximum number of SD hops $\varphi_{0}$ is a constant independent of the number of nodes $n$. Clearly we have $f_{L}(l)=\omega(l)$ and $\lim _{\varphi \rightarrow \infty} \mathrm{E}[L] \leq \lim _{\varphi \rightarrow \infty} \varphi_{0}$ $<\infty$. Therefore, we conclude that the network throughput achieved in this case is scalable.

Example 3: $\lambda(l)=\lambda_{0}\left(1-p_{1}\right)^{l-1} p_{1} / l, \quad p_{1}$ is a constant independent of the number of nodes $n$.

The distribution of SD-distance $f_{L}(l)$ in this case is given by $f_{L}(l)=\frac{1}{l}\left(1-p_{1}\right)^{l-1} / \sum_{l=1}^{\varphi} \frac{1}{l}\left(1-p_{1}\right)^{l-1}$. Instead of deriving $\lim _{\varphi \rightarrow \infty} \mathrm{E}[L]$, an easier way is to apply Theorem 1. In particular, for any $M \geq 1, \lim _{\varphi \rightarrow \infty} \sum_{l=1}^{M} l \lambda(l)=\lim _{\varphi \rightarrow \infty} \theta\left(1-\left(1-p_{1}\right)^{M}\right)>0$. 
Therefore, we conclude that the network throughput achieved in this case is scalable.

\section{B. Optimal Rate Allocation}

Efficient and fair resource allocation among SD pairs is another important issue in network design besides the limit constraints. In this subsection, the optimal rate allocation will be investigated with fairness taken into consideration.

According to (17), the network throughput $\lambda$ is maximized when the mean SD-distance $\mathrm{E}[L]=1$, which implies that the packets are only delivered to the nearest neighbors. In this case the maximum network throughput is equal to the local node throughput, i.e., $\lambda^{*}=\theta$.

$\lambda(l)$ can be further optimized when fairness of rates among different SD pairs is included. In particular, both the proportional fairness and max-min fairness are considered here [20]:

i) Proportional fairness:

$$
\max _{\lambda(l)} \sum_{l=1}^{\varphi} \log (\lambda(l)) \quad \text { subject to } \sum_{l=1}^{\varphi} l \lambda(l)=\theta
$$

From (21), it can be derived that $\lambda_{p}^{*}(l)=\theta /(l \varphi), l=1, \ldots, \varphi$, which leads to a network throughput of

$$
\lambda_{p}^{*}=\frac{\theta}{\varphi} \sum_{l=1}^{\varphi} 1 / l \approx \theta \ln \varphi / \varphi .
$$

ii) Max-min fairness:

$$
\max _{\lambda(l)} \min _{l=1, \ldots, \varphi} \lambda(l) \quad \text { subject to } \sum_{l=1}^{\varphi} l \lambda(l)=\theta
$$

It can be derived that $\lambda_{m}^{*}(l)=\frac{2 \theta}{\varphi(\varphi+1)}, l=1, \ldots, \varphi$, which leads to a network throughput of

$$
\lambda_{m}^{*}=2 \theta /(\varphi+1) .
$$

Comparing (22) and (24), we can see that a higher network throughput can be achieved in the proportional fairness case. The physical interpretation is that in the max-min fairness case the long-distance SD pairs require more transmission resources for each unit of traffic, but yield the same amount of input rate as the short-distance ones do. They may exhaust the network resources and therefore drag down the network throughput. On the other hand, $\lambda(l) \sim 1 / l$ implies a window flow control scheme in which the average numbers of backlogged packets of all SD pairs are the same regardless of their SD-distances. Consequently, all the SD pairs (whether long-distance or shortdistance) share the network resources (i.e., the airtimes required to transport the packets) equally, which in turn leads to a higher input rate for the short-distance ones so that the network throughput can be improved.

\section{TRAFFIC SHAPING}

In this section, we will suggest several traffic scaling laws which may shed some lights on the practical network implementations in the future. An example is also offered to show how to execute the traffic shaping scheme.

\section{A. $\quad$ Traffic Scaling Laws}

The traffic scaling law concerns the regulations of routing and access at each node to achieve scalable network throughput and bounded transport delay. We are interested in the scaling laws that govern the network traffic patterns which fulfill the conditions on the distribution of the SD-distance $L$ discussed in Sec. III. A.

In order to comply with these conditions, we offer three sets of traffic scaling laws to ensure that the network scalability can be accomplished by properly choosing the scaling parameters.

1) Power Law Scaling: $f_{L}(l)=c_{0} l^{\alpha}$

2) Exponential Scaling: $f_{L}(l)=c_{0} l^{\alpha} e^{-\beta l}$

3) Normal Scaling: $f_{L}(l)=c_{0} l^{\alpha} e^{-\beta l^{2}}$

Note that the SD-distance $L$ is a discrete random variable representing the number of hops from the source to the destination in the previous sections. Here, for the sake of discussion, we will treat $L$ as a continuous random variable, i.e., the probability density function (pdf) of the SD-distance $L$, $f_{L}(l)$, is a continuous function in the interval $[0, \infty] .{ }^{1}$

Let us take the example of the class of power law distribution. Let $\varepsilon>0$ be the minimum SD-distance. The normalization of $f_{L}(l)$ requires that $\int_{\varepsilon}^{\infty} f_{L}(l) d l=1$, which implies $c_{0}=-(1+\alpha) / \varepsilon^{1+\alpha}$ and $\alpha<-1$. A positive scaling parameter $\alpha>0$ indicates that the probability of selecting a far-away destination is always higher than that of selecting a closer one, while $\alpha=0$ refers to a uniform distribution. We have known from Sec. III. A that both of them cannot lead to a scalable network throughput. It is an easy check that $\mathrm{E}[L]<\infty$ when $\alpha<-2$. This indicates that network scalability can be achieved by the power law distribution with $\alpha<-2$.

\section{B. Leaky-Bucket Scheme}

We have described how to choose the appropriate parameters of the proposed traffic scaling laws. This subsection is devoted to the execution of traffic shaping at the access points of the network in order to comply with those laws.

Fig. 3(a) shows the leaky-bucket traffic shaping scheme implemented at each node. The newly-generated input packets need to get tokens before they are transmitted. Eqn. (17) indicates that both the input rate $\lambda$ and the SD-distance $L$ contribute to the input traffic. Therefore, it requires $L$ tokens to enter the network for a packet with $L$ hops. Note that once the packet entered the network, tokens are no longer needed for its remaining journey in the network. Hence, the relay packets can go directly to the conforming buffer without getting any tokens.

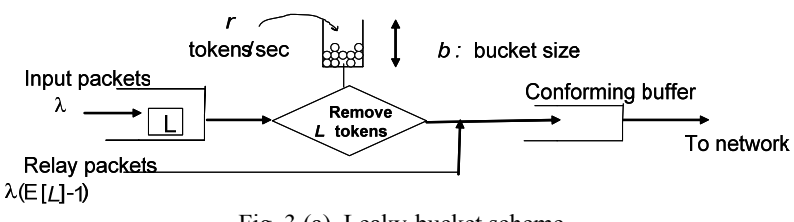

Fig. 3 (a). Leaky-bucket scheme

The tokens are generated at a rate of $r$, which is determined by the local node throughput and bounded by $e^{-1} /\left(\pi R^{2} \sigma\right)$. The bucket size $b$ is the maximum number of tokens in the bucket. Suppose $m$ input packets were conformed in the time interval

\footnotetext{
${ }^{1}$ With the continuous pdf, it is easy to obtain the corresponding probability mass function. For example, the probability of $L=x$ is $\int_{x-1}^{x} f_{L}(l) d l, x=1,2, \ldots$.
} 
$[t, t+\tau]$, and there were $a$ tokens in the bucket at time $t, a \leq b$. We then have

$$
L_{1}+L_{2}+\ldots+L_{m} \leq a+r \tau \Rightarrow \frac{m}{\tau} \cdot \frac{L_{1}+L_{2}+\ldots+L_{m}}{m} \leq r+\frac{a}{\tau}(25)
$$

For large enough $\tau$, (25) implies $\lambda \mathrm{E}[L] \leq r+\varepsilon_{0}$, where $\varepsilon_{0}=a / \tau \leq$ $b / \tau$ is the over provision error.

The bucket size, $b$, is a design parameter related to the efficiency of the leaky-bucket. The bucket size $b$ cannot be too large as the over provision error is bounded by $b$. On the other hand, if $b$ is too small, excessive delay may be incurred. A compromise between error and delay is to set the bucket size between the mean value and an maximum threshold of $L$, i.e., $\mathrm{E}[L] \leq b \leq L_{m}$, where $\operatorname{Pr}\left\{L>L_{m}\right\} \leq q, q$ is a small value. The maximum threshold $L_{m}$ is determined by the distribution $f_{L}(l)$. Take the Rayleigh distribution as an example. It can be obtained that $L_{m} \geq 2 \sqrt{-\ln q / \pi} \cdot \mathrm{E}[L]$. With $q=e^{-6}<0.25 \%, \mathrm{E}[L] \leq b \leq$ $2.764 \mathrm{E}[L]$. Certainly, the bucket size $b$ does not have to be fixed. It can be adjusted dynamically subject to the traffic condition.

In the scheme described above, all new packets obtain the tokens from a common token bucket. If one of them has a large SD-distance $L$, it will exhaust the token bucket and incur excessive delay for the others. To avoid such instance, we further introduce parallel token buckets as shown in Fig. 3(b). The new packets are assigned to different queues according to the SD-distance $L$, i.e., the packets with the same number of hops wait at the same queue. A token bucket is designated to each queue, and the packets can obtain tokens only from their own token bucket.

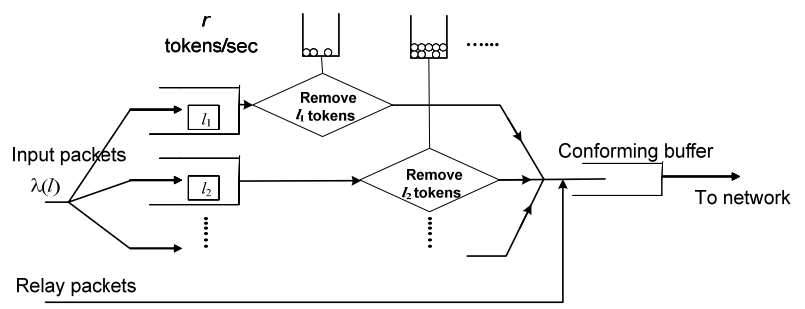

Fig. 3 (b). Leaky-bucket scheme with parallel token buckets

The tokens are still generated at a rate of $r$. However, we can adopt different rules to allocate the tokens. For example, if the tokens are equally allocated to each token bucket, the input rate of the packets with a larger SD-distance $L$ will be lower, because they consume more tokens at each time. This actually implies a rate allocation of $\lambda(l) \sim 1 / l$. On the other hand, if the tokens are allocated proportionally to the SD-distance $L$, the input rate of all the packets will be the same, i.e., $\lambda(l)=\lambda_{0}$. Clearly in the latter case, the packets with a larger $L$ occupy more network resources. As shown in Sec. III. B, these two rate allocation schemes actually correspond to the proportional fairness and max-min fairness, respectively. The max-min fairness case will lead to a lower network throughput.

\section{CONCLUSIONS}

In this paper, we have derived the network throughput and transport delay based on a statistical wireless network model. We demonstrate that the network traffic is determined by both the input rate and the distribution of the SD distance. The necessary and sufficient condition for scalable network throughput shows that the network scalability depends critically on how local the traffic is.

The issue of wireless networking is not just the overall throughput in general, but also which SD pair gets what throughput and the related fairness consideration. We address these issues by formulating a resource-allocation problem and the optimal rate allocation is investigated with fairness taken into consideration.

We conclude this paper with an illustration of how our theories may be applied in practice. We provide several traffic scaling laws and show how the different parameters in leakybucket traffic shaping can be configured to realize the desirable characteristics as indicated by our theories.

\section{REFERENCES}

[1] S. Verdu, "Fifty years of Shannon Theory," IEEE Trans. Inf. Theory, vol. 44, no. 6, pp. 2057-2078, Oct. 1998

[2] A. Ephremides and B. Hajek, "Information theory and communication networks: an unconsummated union," IEEE Trans. Inf. Theory, vol. 44, no. 6, pp. 2416-2434, Oct. 1998.

[3] L. Tong, V. Naware and P. Venkitasubramaniam, "Signal processing in random access," IEEE Signal Processing Magazine, vol. 21, no. 5, pp. 29-39, Sept. 2004.

[4] A. Alwan, R. Bagrodia, N. Bambos, M. Gerla, L. Kleinrock, J. Short and J. Villasenor, “Adaptive mobile multimedia networks," IEEE Personal Commun. Mag., vol. 3, no.2, pp. 34-51, Apr. 1996.

[5] P. Gupta and P. R. Kumar, "The capacity of wireless networks," IEEE Trans. Inf. Theory, vol. 46, no. 2, pp. 388-404, Mar. 2000.

[6] J. Li, C. Blake, D. S. J. De Couto, H. I. Lee and R. Morris, "Capacity of ad hoc wireless networks," in Proc. ACM MobiCom 2001.

[7] M. Franceschetti, O. Dousse, D. N. C. Tse, and P. Thiran, "Closing the gap in the capacity of wireless networks via percolation theory," IEEE Trans. Inf. Theory, vol. 53, no. 3, pp. 1009-1018, Mar. 2007.

[8] O. Dousse and P. Thiran, "Connectivity vs. capacity in dense ad hoc networks," in Proc. Infocom 2004.

[9] A. Jovicic, P. Viswanath and S. R. Kulkarni, "Upper bounds to transport capacity of wireless networks," IEEE Trans. Inf. Theory, vol. 50, no. 11, pp. 2555-2565, Nov. 2004.

[10] L.-L Xie and P. R. Kumar, "A network information theory for wireless communication: scaling laws and optimal operation," IEEE Trans. Inf. Theory, vol. 50, no. 5, pp. 748-767, May 2004.

[11] O. Leveque and E. Telatar, "Information-theoretic upper bounds on the capacity of large extended ad hoc wireless networks," IEEE Trans. Inf. Theory, vol. 51, no. 3, pp. 858-865, Mar. 2005.

[12] M. Grossglauser and D. Tse, "Mobility increases the capacity of ad hoc wireless networks," IEEE/ACM Trans. Networking, vol. 10, no. 4, pp. 477-486, Aug. 2002.

[13] S. Toumpis and A. J. Goldsmith, "Large wireless networks under fading, mobility and delay constraints," in Proc. Infocom 2004.

[14] A. E. Gamal, J. Mammen, B. Prabhakar, and D. Shah, "Throughput delay trade-off in wireless networks," in Proc. Infocom 2004.

[15] G. Sharma, R. Mazumdar, N. Shroff, "Delay and capacity trade-offs in mobile ad hoc networks: a global perspective," in Proc. Infocom 2006.

[16] A. Ozgur, O. Leveque and D. N. C. Tse, "Hierarchical cooperation achieves optimal capacity scaling in ad hoc networks," to appear in IEEE Trans. Inf. Theory.

[17] F. Baccelli, B. Blaszczyszyn and P. Muhlethaler, "An Aloha protocol for multihop mobile wireless networks," IEEE Trans. Inf. Theory, vol. 52, pp. 421-436, Feb. 2006.

[18] Edward P. C. Kao, An Introduction to Stochastic Processes. Duxbury Press, 1996.

[19] Leonard Kleinrock, Queueing Systems. Hohn Wiley \& Sons, 1975.

[20] F. P. Kelly, A. K. Maulloo and D. K. H. Tan, "Rate control in communication networks: shadow prices, proportional fairness and stability," Journal of the Operational Research Society, vol. 49, pp. 237$252,1998$. 